\section{EPIDEMIOLOGY OF ILLNESS IN FEMALE ATHLETES: A SYSTEMATIC REVIEW}

${ }^{1}$ Thomas Axon, $23,4,5$ Nirmala Perera. ${ }^{1}$ Royal College of General Practitioners, UK; ${ }^{2}$ Sport Without Injury ProgrammE (SWIPE), Division of Physiotherapy, Department of Medical and Health Sciences, Linköping University, Linköping, Sweden; ${ }^{3}$ Nuffield Department of Orthopaedics, Rheumatology, and Musculoskeletal Sciences, University of Oxford, Oxford, UKi ${ }^{4}$ Centre for Sport, Exercise and Osteoarthritis Research Versus Arthritis, UK; ${ }^{5}$ School of Allied Health, College of Science, Health and Engineering, Latrobe University, Melbourne, Australia

\subsection{6/bjsports-2021-IOC.217}

Background Illness prevention within elite sport can confer performance and health benefits to athletes. Despite the growing popularity and coverage of women's sport, little is currently known about the illness profiles of female athletes.

Objective To describe the incidence and nature of illness in female athletes.

Design This systematic review was prospectively registered (PROSPERO CRD42018092373). CINAL, Medline, PsychINFO, PubMed, SPORTDiscus were systematically searched from January 2008 to March 2018 inclusively. Peer-reviewed original research articles which reported the incidence and nature of illness in elite female athletes aged $18+$ years were included. The risk of bias was assessed independently by two authors using the STROBE Statement Rating Criteria for systematic reviews. The included studies were mapped against the NHMRC evidence hierarchy.

Main Outcome Measurements Incidence and nature of acute and chronic illness.

Results 118 studies met the inclusion criteria; including 65 cohort studies (level ii/iii) and 46 cross-sectional studies (level iv evidence). The mean risk of bias was 16/22 (median=16, range 8-21). Of the included studies, 66 were in elite athletes (including 14 studies in Olympians), and 40 studies investigated collegiate/university athletes. Infections (primarily respiratory and gastrointestinal) were the most frequent cause of illness (46\%), followed by mental health issues $(20 \%)$, regardless of the sport or setting. Heterogeneity of illness definitions, severity classifications, and inconsistencies in exposure measures prevented any direct comparisons of illness incidence/severity across levels of competition.

Conclusions Illness prevention in women's sport is a novel and emerging research area. Currently there is a lack of consensus on illness data collection, and the majority of studies did not report important methodological information such as participant characteristics, or loss to follow-up. Owing to the lack of good-quality data, the findings are only able to provide a partial overview of the illness profile of female athletes, limiting the development of prevention interventions.

\section{ANTHROPOMETRICS AND KNEE DYNAMIC VALGUS AS EXPLANATORY FACTORS FOR LANDING KINETICS AND ACL INJURY RISK: A FEMALE FOOTBALL COHORT STUDY}

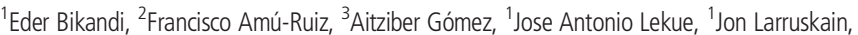
${ }^{1}$ Xabier Monasterio, ${ }^{1}$ Julen Arce, ${ }^{3,4}$ Igor Setuain, ${ }^{4}$ Mikel Izquierdo. ${ }^{1}$ Athletic Club. Medical Department, Bilbao, Spain; 'Universidad del Valle. Department of Sports Science, Cali, Colombia; ${ }^{3}$ Traumatología Deportiva Navarra, Pamplona, Spain; ${ }^{4}$ Universidad Pública de Navarra, Pamplona, Spain
}

$10.1136 /$ bjsports-2021-IOC.218
Background Due to the devastating impact of the anterior cruciate ligament (ACL) injury has on the female athlete's career, anthropometrics and biomechanics-related risk factor screenings have been widely designed. However, the relation of aberrant movement patterns and other anthropometrical variables still remains controversial.

Objective The aim of this study was to analyse if lower limb anthropometrics and knee kinematics could explain landing kinetics during a drop jump maneuver, as primary ACL injury risk factors in a female football environment.

Design Prospective cross-sectional descriptive study. Testing methodology was settled inside an anatomical, biomechanical and functional conditioning risk factor screening. Data collection was made during the pre-season period.

Setting 4 different teams from the region of the Basque Country (Spain). 2 youth second teams and 2 senior teams competing in the Spanish second division.

Patients (or Participants) 64 youth female football players (age 20,32 $\pm 4,9$ ). Every available (non-injured) athlete was included. No lost participants during the data collection.

Interventions (or Assessment of Risk Factors) Pelvic width and femur length were measured as anthropometrical gold variables. Knee valgus kinematics and landing kinetics during the bilateral and unilateral drop jump were registered.

Main Outcome Measurements Knee valgus kinematic data (femur-tibia angle $<180^{\circ}$ ) was captured by a video analysis tracker software. Landing kinetics were registered using inertial sensor unit technology.

Results There was a little correlation $(r=0,303)$ between landing peak VGRF and knee valgus kinematics, at $\mathrm{p}=0,01$ significance level, and also with the femur length $(r=-0,338)$. In addition, the linear regression analysis showed that the pelvic width, femur length and knee valgus kinematics explained in $89,1 \%\left(R^{2}\right)$ the landing kinetics results $(p=<0,05)$.

Conclusions A moderate significance level between lower limb anthropometrics, knee valgus kinematics and landing kinetics was the main finding at the present study. More exhaustive surveillance screening re-tests are needed in order to detect the main contributors for ACL injury mechanics in a female football context.

\section{CONTACT INJURIES PREDOMINATE IN FEMALE YOUTH TEAM SPORTS: AN OPPORTUNITY FOR PREVENTION}

${ }^{1}$ Alexis Cairo, ${ }^{1}$ Isla Shill, ${ }^{1}$ Anu Räisänen, ${ }^{1,2,3,4}$ Amanda Black, $1,2,3,4,5,6,7$ Carolyn Emery. ${ }^{1}$ Sport Injury Prevention Research Centre, Faculty of Kinesiology, University of Calgary, Calgary, Canada; ${ }^{2}$ Alberta Children's Hospital Research Institute, University of Calgary, Calgary, Canada; ${ }^{3} \mathrm{O}^{\prime} B r i e n$ Institute for Public Health, University of Calgary, Calgary, Canada; ${ }^{4}$ Hotchkiss Brain Institute, University of Calgary, Calgary, Canada; ${ }^{5}$ McCaig Institute for Bone and Joint Health, University of Calgary, Calgary, Canada; ${ }^{6}$ Community Health Sciences, Cumming School of Medicine, University of Calgary, Calgary, Canada; ${ }^{6}$ Community Health Sciences, Cumming School of Medicine, University of Calgary, Calgany, Canada; 'Department of Pediatrics, Cumming School of Medicine, University of Calgary, Calgary, Canada

\subsection{6/bjsports-2021-IOC.219}

Background Sport participation is the leading cause of injury in youth. Female youth are underrepresented in the sport injury literature and present with a different injury profile than males.

Objective To investigate sport-related injury rates, types, locations and mechanisms in female youth team sports. Design Secondary analysis of a cross-sectional study. 
Setting High schools in Alberta, Canada.

Participants Female students who reported playing one of the top ten team sports for participation (i.e., baseball, basketball, lacrosse, soccer, volleyball, football, rugby, ringette, field hockey, ice hockey).

Assessment of Risk Factors

A 45-minute survey included questions regarding demographic information, sport participation, and one-year injury and concussion history.

Main Outcome Measurements Self-reported injuries sustained in the past year.

Results 51.7\% (1048/2029) of high school students completing the survey were female and 481/1048 (45.9\%) reported playing at least one team sport. Of these, $51.4 \%$ reported at least one sport-related injury and $8.9 \%$ at least one concussion in the past year. Injury rate based on 'most serious injury' reported was highest in ringette (42.9 injuries/100 students/ year) and rugby (40.0). The top three most serious injury locations were the knee (24.7\%), ankle (21.6\%) and head (16.1\%). The most common injury types were joint or ligament sprain (26.7\%), fracture (13.0\%) and concussion $(11.8 \%)$. Based on all serious injuries reported in female team sports, $73.4 \%$ occurred via contact mechanisms (with someone or something). Overuse (16.2\%) was the next most common mechanism reported. Of participants that reported concussion as their most serious injury, $100 \%$ were attributed to contact mechanisms $(38.9 \%$ contact with someone; $61.1 \%$ contact with something).

Conclusions Team sport injury rates are high in female high school students. Specific consideration of contact injury mechanisms in female youth team sports will inform development and evaluation of targeted female contact and sport-specific prevention strategies.

\section{PREVALENCE OF MENSTRUAL DISORDERS AMONG GERMAN FEMALE ELITE ATHLETES}

1,2Elisabeth M Kirschbaum, ${ }^{3}$ Katharina Fischer, ${ }^{4}$ Jan C Wuestenfeld, ${ }^{4,5}$ Bernd Wolfarth. ${ }^{1}$ Department of Game and Combat Sports, Institute for Applied Training Science, Leipzig, Germany; ${ }^{2}$ Department of Sport and Health Sciences, Biomechanics in Sports, Technical University of Munich, Munich, Germany; ${ }^{3}$ Department of Endurance, Institute for Applied Training Science, Leipzig, Germany; ${ }^{4}$ Department of Sports Medicine, Institute for Applied Training Science, Leipzig, Germany; ${ }^{5}$ Department of Sports Medicine, Charité University School of Medicine, Humboldt University, Berlin, Germany

\section{$10.1136 /$ bjsports-2021-IOC.220}

Background A healthy menstrual cycle affects the athletic performance in training and competition. However, the prevalence of menstrual cycle disorders among elite athletes is unclear.

Objective To assess the prevalence of menstrual disorders among German female elite athletes in 2019 and 2020.

Design Retrospective cohort study.

Setting German female youth and adult elite athletes.

Participants 532 female elite athletes $(19.5 \pm 5.0$ years; 170.0 $\pm 7,3 \mathrm{~cm} ; 62.1 \pm 11.4 \mathrm{~kg}$ ) were included in this study. All athletes were members of the German funding for high performance sports.

Assessment of Risk Factors Menstrual disorder, sport discipline, age, BMI, body fat, training volume.

Main Outcome Measurements All athletes completed a baseline gynaecological survey. The independent samples t-test and ANOVA were used for statistical analysis $(p<.05)$.
Results 118 of all 532 of the female athletes (22.2\%) had an irregular menstrual cycle. The most prevalent menstrual disorders are oligomenorrhea (41.9\%), secondary amenorrhea (25.0\%), primary amenorrhea (11.3\%), dysmenorrhea (4.0\%), metrorrhagia (2.4\%), hypermenorrhea (1.6\%), polymenorrhea $(0.8 \%)$ and others $(12.9 \%)$. There is a significant difference in the prevalence of menstrual disorders among sport disciplines $(\mathrm{p}=.000, \mathrm{f}=.214)$. The highest prevalence of menstrual disorders occurs among endurance sport athletes (30.9\%). Athletes with menstrual disorders have a significantly lower BMI $(\mathrm{p}=.014, \mathrm{~d}=.258)$ and lower percent body fat $(\mathrm{p}=.000$, $\mathrm{d}=.392$ ) compared to athletes with normal menstrual cycles. There is no significant difference in age $(p=.101, d=.172)$ and training volume $(\mathrm{p}=.100, \mathrm{~d}=.172)$ between the groups.

Conclusions Our research showed a high prevalence of menstrual disorders among German female elite athletes. The results suggest that especially athletes with low BMI and low percent body fat are at a high risk for menstrual disorders. Further research is required to investigate the effects of menstrual disorders on athletic performance and long-term health.

\section{MONITORING WELLBEING AND PERCEIVED EXERTION IN RELATION TO INJURY RISK IN ELITE FEMALE FOOTBALL PLAYERS OVER 2 SEASONS}

${ }^{1} J o n$ Larruskain, ${ }^{2}$ Ane Uria, ${ }^{1}$ Maialen Aldalur, 'Iraia Iturregi, 'Aritz Yarritu, 'Eder Bikandi, ${ }^{1} J u l e n$ Arce, ${ }^{1}$ Xabier Monasterio, ${ }^{1} J o s e$ Antonio Lekue, ${ }^{1}$ Imanol Martin-Garetxana. ${ }^{1}$ Athletic Club, Medical Services, Lezama, Spain; ${ }^{2}$ University of Glasgow, Glasgow, UK

\subsection{6/bjsports-2021-IOC.221}

Background There is a gap in understanding how to use wellbeing and load measures in football, particularly in women. Objective To describe the association of wellbeing and sessionrating of perceived exertion (sRPE) with injury risk.

Design Prospective cohort study.

Setting Elite football team from the Spanish first division.

Participants 28 elite female players were followed during the 2017-2018 and 2018-2019 seasons.

Assessment of Risk Factors Fatigue, sleep quality, muscle soreness, stress and mood (questionnaire from 1-worst to 5-best), exposure time in training and matches, RPE (0-10) and injuries were daily recorded. Daily and 7-day rolling z-scores were calculated for wellbeing items; and rolling 7 and 28-day sums, uncoupled 7 to 28-day ratios and week-to-week changes for sRPE [time $\mathrm{x}$ RPE in arbitrary units (au)]. In addition, the median sRPE of each session was estimated to account for the load of sessions where injuries occurred.

Main Outcome Measurements The association of wellbeing and sRPE variables with non-contact injuries requiring 4 or more days of absence occurring on a given session was investigated using linear mixed models.

Results The probability of injury was higher in matches $(1.36 \%)$ than in training $(0.44 \%, p=0.001)$. In training, sessions with high median sRPE $(>525$ au, 0.87 vs. the rest $0.35 \%$ ), high 7 -day loads (>2095 au, 0.76 vs. $0.31 \%$ ), low 28 -day loads ( $<5020$ au, 0.71 vs. $0.32 \%)$ and worse than usual (<-1 z-score) 7 -day fatigue $(0.82$ vs. $0.35 \%)$ and muscle soreness (1.19 vs. $0.28 \%)$ were associated with higher injury risk $(\mathrm{p}<0.05)$. Moreover, pre-match worse than usual daily sleep quality (3.03 vs. $0.85 \%)$, stress (4.55 vs. $0.92 \%)$ and mood $(2.74$ vs. $0.95 \%)$ showed higher injury probabilities $(p<0.05)$. Interactions between wellbeing and sRPE were also observed. 